\title{
Systems of singular differential equations with pulse action
}

\author{
Alexandr Boichuk', Martina Langerová², Miroslava Růžičková2* and Evgenij Voitushenko³
}

${ }^{*}$ Correspondence:

miroslava.ruzickova@fhv.uniza.sk

${ }^{2}$ University of Žilina, Žilina, Slovakia

Full list of author information is

available at the end of the article

\begin{abstract}
The paper deals with the singular systems of ordinary differential equations with impulsive action under the assumption that the considered systems can be reduced into the central canonical form. An approach which combines the theory of impulsive differential equations and known results from the theory of singular Fredholm boundary value problems is used. Necessary and sufficient conditions for the existence of solutions of the singular boundary value problems with impulsive action are derived. Moreover, an algorithm for the construction of the family of linearly independent solutions is shown.

MSC: 34A09; 34A37
\end{abstract}

Keywords: singular systems; impulsive action; central canonical form; existence of solutions; Moore-Penrose pseudo-inverse matrix

\section{Introduction}

It is known that some of the problems of the control theory, radio physics, mathematical economics, linear programming and others can be modeled by systems of differential equations with a singular matrix. Such systems, which are the so-called singular or differential-algebraic systems of ordinary differential equations, are studied in many works; in addition to other contributions, for example, in [1-3]. Campbell and Petzold have introduced in [1] the so-called central canonical form of singular linear systems which plays a very important role in the study of such problems. The reduction to the central canonical form is already the classical condition for this type of singular or differentialalgebraic problems [4].

The existence of solutions of problems with singular matrix, mainly initial-value and periodic problems, was studied in [5]. This paper deals with the most complicated and the least studied resonance problems [6] for singular differential systems with impulsive action. The origin of the theory of differential systems with impulsive action can be found in the work by Myshkis and Samoilenko [7], later also in the work by Samoilenko and Perestyuk [8]. The theory was also developed by Halanay and Wexler [9], Schwabik et $a l$. [10] and others. The ideas proposed in these works have been further developed and generalized in numerous other publications; for example, in [11-13].

The main aim of this contribution is to establish necessary and sufficient conditions for the existence of solutions of the singular systems of ordinary differential equations with impulsive action in a relevant space. In order to do so, we will use the theory of impulsive differential equations and known results from the theory of singular Fredholm boundary 
value problems [6]. Moreover, an algorithm for finding solutions of such problems in the general case under the assumption that the unperturbed singular differential systems can be reduced into the central canonical form is suggested in the paper.

Let us consider the problem of existence and construction of solutions of the singular linear systems of ordinary differential equations with impulsive action at fixed points of time

$$
\begin{aligned}
& B(t) \dot{x}=A(t) x+f(t), \quad t \in[a, b], \\
& \left.\Delta E_{i} x\right|_{t=\tau_{i}}=S_{i} x\left(\tau_{i}-0\right)+\gamma_{i}, \quad \tau_{i} \in(a, b), i=1, \ldots, p,
\end{aligned}
$$

where $A(t), B(t)$ are $n \times n$ matrices, $\operatorname{det} B(t)=0, \forall t \in[a, b] ; f(t)$ is an $n$-dimensional column vector function; $E_{i}, S_{i}$ are $m_{i} \times n$ real constant matrices; $\gamma_{i}, i=1, \ldots, p$, are $m_{i}$-dimensional real constant column vectors, i.e., $\gamma_{i} \in \mathbb{R}^{m_{i}}$, and $\left.\Delta E_{i} x\right|_{t=\tau_{i}}:=E_{i}\left(x\left(\tau_{i}+\right)-x\left(\tau_{i}-\right)\right)$.

We suppose that the components of the matrices $A(t), B(t)$ and the vector $f(t)$ are real sufficiently many times continuously differentiable functions on the interval $[a, b]$. The property 'sufficiently many times' depends on the rank of the matrix $B(t)$, as will be seen below.

The solution $x(t)$ is being sought in the space of $n$-dimensional piecewise continuously differentiable vector functions, $x(t) \in C^{1}\left([a, b] \backslash\left\{\tau_{1}, \ldots, \tau_{p}\right\}\right)$.

The norms in the spaces $C\left([a, b] \backslash\left\{\tau_{1}, \ldots, \tau_{p}\right\}\right), C^{1}\left([a, b] \backslash\left\{\tau_{1}, \ldots, \tau_{p}\right\}\right)$ are introduced in the standard manner, by analogy with $[8,9]$.

Note that if

1. $\forall i=1, \ldots, p$ are $E_{i}:=E n \times n$ identity matrices, $S_{i} n \times n$ matrices and hence $\gamma_{i}$ $n$-dimensional column vectors, then the conditions (2) take the form of the standard impulsive conditions (see in [8]):

$$
\left.\Delta x\right|_{t=\tau_{i}}:=x\left(\tau_{i}+\right)-x\left(\tau_{i^{-}}\right)=S_{i} x\left(\tau_{i}^{-}\right)+\gamma_{i}, \quad i=1, \ldots, p .
$$

2. $E_{1}:=(1,0, \ldots, 0), \ldots, E_{i}:=(0, \ldots, 0,1,0, \ldots, 0), \ldots, i=1, \ldots, p, p \leq n$ and $S_{i}$ are $1 \times n$ vectors, then the conditions (2) take the form of impulsive conditions only on the corresponding components of the vectors $x(t)=\operatorname{col}\left(x_{1}(t), \ldots, x_{i}(t), \ldots, x_{n}(t)\right)$ :

$$
\left.\Delta E_{i} x\right|_{t=\tau_{i}}:=x_{i}\left(\tau_{i}+\right)-x_{i}\left(\tau_{i}^{-}\right), \quad i=1, \ldots, p .
$$

\section{Auxiliary results}

We have already mentioned that the reducibility of singular linear systems to the central canonical form is the key issue in the study of singular problems. Some useful results related to these problems are proved in [5]. Here these results are formulated into an auxiliary statement in the form of Lemma 1 , and they will be further used to solve the problem (1), (2).

Let us introduce the homogeneous system

$$
B(t) \frac{d x}{d t}=A(t) x, \quad t \in[a, b]
$$

associated to system (1) and the corresponding adjoint system

$$
\frac{d}{d t} B^{*}(t) y=-A^{*}(t) y, \quad t \in[a, b]
$$

to system (3). 
Let $X_{n-s}(t)$ be an $n \times(n-s)$ matrix formed by $n-s$ linearly independent solutions of system (3) and let $Y_{n-s}(t)$ be an $n \times(n-s)$ matrix formed by $n-s$ linearly independent solutions of adjoint system (4).

The matrices $X_{n-s}(t)$ and $Y_{n-s}(t)$ are called fundamental matrices of systems (3) or (4) respectively. In addition to this, we suppose that the fundamental matrices $X_{n-s}(t), Y_{n-s}(t)$ are constructed (see in $[5, \mathrm{p} .64])$ so as to ensure that

$$
Y_{n-s}^{*}(t) B(t) X_{n-s}(t)=E_{n-s}
$$

Lemma 1 Let us assume that the following conditions are satisfied:

(i) $\operatorname{rank} B(t)=n-r_{1}$ for all $t \in[a, b]$;

(ii) there exists a complete Jordan set of vectors $\varphi_{i}^{j}(t), j=1, \ldots, s_{i}, i=1, \ldots, r_{1}$, of the matrix $B(t)$ on the given interval $[a, b]$ with respect to the operator $L(t)=A(t)-B(t) \frac{d}{d t}$, which is determined by the relations

$$
\begin{aligned}
& B(t) \varphi_{i}^{1}(t)=o, \\
& B(t) \varphi_{i}^{j}(t)=L(t) \varphi_{i}^{j-1}(t), \quad j=2, \ldots, s_{i}, i=1, \ldots, r_{1}
\end{aligned}
$$

(iii) $A(t), B(t) \in C^{3 q-2}[a, b] ; f(t) \in C^{q-1}[a, b], q=\max _{i} s_{i}$.

Then

(1) there exist matrices $P(t), Q_{1}(t) \in C^{q-1}[a, b]$ which are nonsingular for all $t \in[a, b]$ and such that the multiplication by $P(t)$ and the substitution $x=Q_{1}(t) y$ reduce system (1) to the central canonical form

$$
\left(\begin{array}{cc}
E_{n-s} & 0 \\
0 & I
\end{array}\right) \frac{d y}{d t}=\left(\begin{array}{cc}
M(t) & 0 \\
0 & E_{s}
\end{array}\right) y+P(t) f(t)
$$

where $E_{s}, E_{n-s}, s=s_{1}+s_{2}+\cdots+s_{r_{1}}$, are the $s \times s$ or $(n-s) \times(n-s)$ respectively identity matrices, $I=\operatorname{diag}\left(I_{1}, \ldots, I_{r_{1}}\right)$ is the quasi-diagonal matrix consisting of nilpotent Jordan blocks $I_{i}, i=1, \ldots, r_{1}$, of orders $s_{i}$.

(2) there exists the general solution of system (1) in the form

$$
x(t, c)=X_{n-s}(t) c+\widetilde{x}(t)
$$

on the interval $[a, b]$

It is necessary to clarify that $c$ in (6) is an arbitrary $(n-s)$-dimensional constant vector and $\tilde{x}(t)$ is a partial solution of the nonhomogeneous system (1) of the form

$$
\tilde{x}(t)=\int_{a}^{t} X_{n-s}(t) Y_{n-s}^{*}(\tau) f(\tau) d \tau-\Phi(t) \sum_{k=0}^{q-1} \mathrm{I}^{k} \frac{d^{k}}{d t^{k}}\left(\left[\Psi^{*}(t) L(t) \Phi(t)\right]^{-1} \Psi^{*}(t) f(t)\right)
$$

where $\Phi(t), \Psi(t)$ are the $n \times s$ matrices consisting of vectors that form the abovementioned Jordan sets,

$$
\begin{aligned}
& \Phi(t):=\left[\varphi_{1}^{(1)}(t), \ldots, \varphi_{1}^{\left(s_{1}\right)}(t) ; \varphi_{2}^{(1)}(t), \ldots, \varphi_{2}^{\left(s_{2}\right)}(t) ; \ldots ; \varphi_{r_{1}}^{(1)}(t), \ldots, \varphi_{r_{1}}^{\left(s_{r_{1}}\right)}(t)\right], \\
& \Psi(t):=\left[\psi_{1}^{\left(s_{1}\right)}(t), \ldots, \psi_{1}^{(1)}(t) ; \psi_{2}^{\left(s_{2}\right)}(t), \ldots, \psi_{2}^{(1)}(t) ; \ldots ; \psi_{r_{1}}^{\left(s_{r_{1}}\right)}(t), \ldots, \psi_{r_{1}}^{(1)}(t)\right] .
\end{aligned}
$$


Note, it follows from condition (ii) that there exists a similar Jordan set of vectors $\psi_{i}^{j}(t), j=1, \ldots, s_{i}, i=1, \ldots, r_{1}$, of the adjoint matrix $B^{*}(t)$ with respect to the operator $L^{*}(t)=A^{*}(t)+\frac{d}{d t} B^{*}(t)$. The vectors that form these sets are linearly independent for all $t \in[a, b]$, and the completeness condition implies that the determinant consisting of inner products of the vectors $L \varphi_{i}^{\left(s_{i}\right)}(t)$ by basis elements of the null space of the matrix $B^{*}(t)$ is nonzero, i.e., $\operatorname{det}\left\|\left(L(t) \varphi_{i}^{\left(s_{i}\right)}(t), \psi_{i}^{j}(t)\right)\right\|_{i, j=1, \ldots, r_{1}} \neq 0$.

\section{Main results}

In the first part of this section, the relationship of the considered problem (1), (2) with an interface (see in [14]) boundary value problem is shown and the solvability conditions of these problems are derived. Then, in the second part, the case when the solvability conditions are not satisfied is discussed.

\subsection{Connection with the interface boundary value problem}

Using the following notations:

$$
\left\{\begin{array}{l}
\ell_{1} x:=E_{1} x\left(\tau_{1}+\right)-\left(E_{1}+S_{1}\right) x\left(\tau_{1}-\right), \\
\ell_{2} x:=E_{2} x\left(\tau_{2}+\right)-\left(E_{2}+S_{2}\right) x\left(\tau_{2}-\right), \\
\vdots \\
\ell_{p} x:=E_{p} x\left(\tau_{p}+\right)-\left(E_{p}+S_{p}\right) x\left(\tau_{p}-\right),
\end{array}\right.
$$

the systems of singular differential equations with pulse action (1), (2) can be modified to the following equivalent interface boundary value problem:

$$
\begin{aligned}
& B(t) \dot{x}=A(t) x+f(t), \quad t \in[a, b], \\
& \ell x(\cdot)=\gamma,
\end{aligned}
$$

where $\gamma:=\operatorname{col}\left(\gamma_{1}, \ldots, \gamma_{m_{p}}\right) \in \mathbb{R}^{m}, m:=m_{1}+\cdots+m_{p} ; \ell$ is an $m$-dimensional linear vector functional $\ell:=\operatorname{col}\left(\ell_{1}, \ell_{2}, \ldots, \ell_{p}\right): C^{1}\left([a, b] \backslash\left\{\tau_{1}, \ldots, \tau_{p}\right\}\right) \rightarrow \mathbb{R}^{m} ; \ell_{i}: C^{1}\left([a, b] \backslash\left\{\tau_{i}, \ldots, \tau_{p}\right\}\right) \rightarrow$ $\mathbb{R}^{m_{i}}, i=1,2, \ldots, p$.

The solution $x(t)$ of the problem (8), (9) and hence the solution of the initial problem (1), (2) of singular linear systems of ordinary differential equations with the impulsive action at fixed points of time are sought in the space $C^{1}\left([a, b] \backslash\left\{\tau_{1}, \ldots, \tau_{p}\right\}\right)$ of $n$-dimensional piecewise continuously differentiable vector functions with discontinuities of the first kind at $t=\tau_{i}$. We use the general solution (6),

$$
x(t, c)=X_{n-s}(t) c+\widetilde{x}(t)
$$

of the singular differential system (1) to find the solvability condition and the form of the general solution of the linear nonhomogeneous interface boundary value problem (8), (9) and hence the solution of the problem (1), (2) of singular linear systems of ordinary differential equations with the impulsive action at fixed points of time. The solution (6) is a solution of the boundary value problem (8), (9) if and only if it satisfies the boundary conditions (9). This means that the following algebraic system:

$$
Q c=\gamma-\ell \widetilde{x}(\cdot)
$$


has to be solvable concerning $c \in \mathbb{R}^{n-s}$, where $Q$ is an $m \times(n-s)$ constant matrix,

$$
Q:=\operatorname{col}\left(-S_{1} X_{n-s}\left(\tau_{1}\right), \ldots,-S_{p} X_{n-s}\left(\tau_{p}\right)\right)
$$

The algebraic system (10) is solvable if and only if the right-hand side belongs to the orthogonal complement $N\left(Q^{*}\right)=R(Q)$ of the kernel $N\left(Q^{*}\right)=\operatorname{ker} Q^{*}$ of the adjoint matrix $Q^{*}$, i.e., if the following condition is satisfied:

$$
P_{Q^{*}}\{\gamma-\ell \widetilde{x}(\cdot)\}=o,
$$

where $P_{Q^{n}}:=E_{m}-Q Q^{+}$is an $m \times m$ matrix (an orthogonal projection) projecting the space $\mathbb{R}^{m}$ onto the $\operatorname{ker} Q^{*}$. Let $\operatorname{rank} Q:=n_{1}<n-s$. Since $\operatorname{rank} P_{Q^{*}}=d=m-n_{1}$, by $P_{Q_{d}^{*}}$ we denote a $d \times m$ matrix consisting of $d$ linearly independent rows of the $m \times m$ matrix $P_{Q^{n}} ; Q^{+}$is the unique $(n-s) \times m$ Moore-Penrose pseudoinverse matrix of the matrix $Q$. As a result, the criterion (12) consists of $d$ linearly independent conditions,

$$
P_{Q_{d}}\{\gamma-\ell \widetilde{x}(\cdot)\}=o
$$

It is well known (see [11]), that system (10) has an $r$-parametric family of linearly independent solutions

$$
c=Q^{+}\{\gamma-\ell \widetilde{x}(\cdot)\}+P_{Q_{r}} c_{r}, \quad \forall c_{r} \in \mathbb{R}^{r},
$$

where $P_{Q}:=E_{n-s}-Q^{+} Q$ is an $(n-s) \times(n-s)$ matrix (an orthogonal projection) projecting the space $\mathbb{R}^{n-s}$ onto the $\operatorname{ker} Q$. Since $\operatorname{rank} P_{Q}=r=(n-s)-n_{1}$, by $P_{Q_{r}}$ we denote an $(n-$ $s) \times r$ matrix consisting of $r$ linearly independent columns of the matrix $P_{Q}$. Substituting solutions (14) in expression (6), we get that the singular linear nonhomogeneous boundary value problem (8), (9) has an $r$-parametric family

$$
\begin{aligned}
x\left(t, c_{r}\right)= & X_{n-s}(t) P_{Q_{r}} c_{r}+X_{n-s}(t) Q^{+}\{\gamma-\ell \widetilde{x}(\cdot)\} \\
& +\int_{a}^{t} X_{n-s}(t) Y_{n-s}^{*}(\tau) f(\tau) d \tau \\
& -\Phi(t) \sum_{k=0}^{q-1} \mathrm{I}^{k} \frac{d^{k}}{d t^{k}}\left(\left[\Psi^{*}(t) L(t) \Phi(t)\right]^{-1} \Psi^{*}(t) f(t)\right)
\end{aligned}
$$

of linearly independent solutions if and only if the condition (13) is satisfied. Thus, we have proved the following statement.

Theorem 1 The interface singular boundary value problem (8), (9) and hence the problem (1), (2) are solvable if and only if the nonhomogenities $f(t) \in C^{q-1}([a, b])$ and $\gamma_{i} \in \mathbb{R}^{m_{i}}$ satisfy d linearly independent conditions:

$$
P_{Q_{d}^{*}}\{\gamma-\ell \widetilde{x}(\cdot)\}=o
$$

Moreover, then there exist exactly $r$ linearly independent solutions of the corresponding homogeneous system (3) in the form $x\left(t, c_{r}\right)=X_{r}(t) c_{r}$ and the nonhomogeneous system (1), 
(2) possesses an r-parametric family of linearly independent solutions in the form

$$
x\left(t, c_{r}\right)=X_{r}(t) c_{r}+(G[f, \gamma])(t)
$$

It is obvious, from the formulas (15), (16), that $X_{r}(t)=X_{n-s}(t) P_{Q_{r}}$ is an $n \times r$ matrix and

$$
\begin{aligned}
(G[f, \gamma])(t):= & \int_{a}^{t} X_{n-s}(t) Y_{n-s}^{*}(\tau) f(\tau) d \tau \\
& -\Phi(t) \sum_{k=0}^{q-1} \mathrm{I}^{k} \frac{d^{k}}{d t^{k}}\left(\left[\Psi^{*}(t) L(t) \Phi(t)\right]^{-1} \Psi^{*}(t) f(t)\right) \\
& +X_{n-s}(t) Q^{+} \gamma-X_{n-s}(t) Q^{+} \ell \widetilde{x}(\cdot) .
\end{aligned}
$$

The operator $(G[f, \gamma])(t)$ is usually called the generalized Green operator of the singular boundary value problem (8), (9) which acts on a vector function $f(t) \in C^{q-1}([a, b])$ and $\gamma \in \mathbb{R}^{m}$.

The obtained results in Theorem 1 can be applied to the study of existence solutions of the initial Cauchy problem for singular linear systems of ordinary differential equations

$$
\begin{aligned}
& B(t) \dot{x}=A(t) x+f(t), \quad t \in[a, b], \\
& x(a)=\alpha,
\end{aligned}
$$

where $f(t) \in C^{q-1}[a, b]$ and $\alpha \in \mathbb{R}^{n}$. It is well known that such kind of the initial Cauchy problems with a singular matrix of the system are not solvable for arbitrary $f(t)$ and $\alpha$.

The necessary and sufficient conditions for the existence of solutions of the singular initial Cauchy problem (18), (19) and also the form of the unique solution of this problem directly follow from Theorem 1 as a corollary.

Corollary 1 The initial Cauchy problem (18), (19) for singular linear systems of ordinary differential equations is solvable if and only if the nonhomogenities $f(t) \in C^{q-1}([a, b])$ and $\alpha \in \mathbb{R}^{n}$ satisfy d linearly independent conditions,

$$
P_{D_{d}^{*}}\{\alpha-\tilde{x}(a)\}=o
$$

and possesses a unique solution in the form

$$
x(t)=X_{n-s}(t) D^{+}(\alpha-\tilde{x}(a))+\widetilde{x}(t),
$$

where $D:=X_{n-s}(a)$ is an $n \times(n-s)$ constant matrix, $\operatorname{rank} P_{D^{*}}=d=n-\operatorname{rank} D, P_{D_{d}^{*}}$ is $a d \times n$ matrix consisting of d linearly independent rows of the $n \times n$ matrix $P_{D^{n}}:=E_{n}-D D^{+}(a n$ orthogonal projection) projecting the space $\mathbb{R}^{n}$ onto the coker $D$.

\subsection{Bifurcation conditions}

Our purpose in this part is to determine sufficient conditions for the bifurcation of solutions of the linear singular Fredholm differential system with the impulsive action with a 
small parameter. It will also be assumed that the unperturbed singular differential system can be reduced to the central canonical form.

The crucial assumption in Theorem 1 is the so-called solvability criterion (13). It means, if nonhomogeneities $f \in C^{q-1}[a, b], \gamma_{i} \in \mathbb{R}^{m_{i}}, i=1, \ldots, p$, in the problem (1), (2) are such that (13) is not satisfied, then there exists no solution of the problem. In such a case, we can modify the system (1) by a linear perturbation so that the perturbed singular boundary value problem

$$
\begin{aligned}
& B(t) \dot{x}=A(t) x+f(t)+\varepsilon A_{1}(t) x, \quad t \in[a, b], \\
& \left.\Delta E_{i} x\right|_{t=\tau_{i}}=S_{i} x\left(\tau_{i}-0\right)+\gamma_{i}, \quad \tau_{i} \in(a, b), i=1, \ldots, p,
\end{aligned}
$$

where $A(t), A_{1}(t), B(t) \in C^{3 q-2}[a, b], \operatorname{det} B(t)=0, \gamma_{i} \in \mathbb{R}^{m_{i}}, i=1, \ldots, p, \varepsilon>0$ is a small parameter, will be solvable for any nonhomogeneities. Therefore, it is analyzed whether the problem (1), (2) can be made solvable by introducing linear perturbations and, if this is possible, then of what kind the perturbations $A_{1}(t) \in C^{3 q-2}[a, b]$ should be to make the boundary value problem (22), (23) solvable for all nonhomogeneities $f \in C^{q-1}[a, b]$, $\gamma:=\operatorname{col}\left(\gamma_{1}, \ldots, \gamma_{p}\right) \in \mathbb{R}^{m}$.

Using the Vishik-Lyusternik method and the technique of Moore-Penrose pseudoinverse matrices, an algorithm for finding a family of linearly independent solutions of such problems for the general case can be suggested.

For a simple formulation of the results, it is convenient to use the notation of $d \times r$ matrix $B_{0}$ in the form

$$
\begin{aligned}
B_{0}:= & -P_{Q_{d}^{*}} \ell\left\{\int_{a}^{\cdot} X_{r}(\cdot) Y_{n-s}^{*}(\tau) A_{1}(\tau) X_{r}(\tau) d \tau\right. \\
& \left.-\Phi(\cdot) \sum_{k=0}^{q-1} \mathrm{I}^{k} \frac{d^{k}}{d t^{k}}\left(\left[\Psi^{*}(t) L(t) \Phi(t)\right]^{-1} \Psi^{*}(t) A_{1}(t) X_{r}(t)\right)(\cdot)\right\},
\end{aligned}
$$

where $\ell: C^{1}\left([a, b] \backslash\left\{\tau_{1}, \ldots, \tau_{p}\right\}\right) \rightarrow R^{m}$.

We can formulate the following statement.

Theorem 2 Let us suppose that there exists no solution of the singular generating boundary value problem (1), (2) for some nonhomogeneities $f(t) \in C^{q-1}[a, b], \gamma \in R^{m}$. If

$$
\operatorname{rank} B_{0}=d,
$$

then there exists a $\rho$-parametric ( $\rho:=r-d=n-s-m)$ family of linearly independent solutions of the perturbed singular boundary value problem (22), (23) in the form of a part of the Laurent series in powers of a parameter $\varepsilon$ :

$$
x(t, \varepsilon)=\sum_{i=-1}^{\infty} \varepsilon^{i} x_{i}\left(t, c_{\rho}\right), \quad \forall c_{\rho} \in \mathbb{R}^{\rho},
$$

convergent for fixed $\varepsilon \in\left(0, \varepsilon_{*}\right]$, where $\varepsilon_{*}$ is an appropriate constant characterizing the domain of the convergence of the series (26), and the coefficients $x_{i}\left(t, c_{\rho}\right)$ are determined from the corresponding problems. 
The proof of these results can be done in a similar way as in the works [6, 15-17] and we omit it.

\section{Competing interests}

The authors declare that they have no competing interests.

\section{Authors' contributions}

The authors have made the same contribution. All authors read and approved the final manuscript.

\section{Author details}

${ }^{1}$ National Academy of Science of Ukraine, Kiev, Ukraine. ${ }^{2}$ University of Žilina, Žilina, Slovakia. ${ }^{3}$ Taras Shevchenko National University of Kiev, Kiev, Ukraine.

\section{Acknowledgements}

This research was supported by the Grant No 1/0682/13 of the Grant Agency of the Slovak Republic (VEGA). The authors would like to thank the referees and the editor for helpful suggestions incorporated into this paper.

Received: 19 February 2013 Accepted: 30 May 2013 Published: 26 June 2013

\section{References}

1. Campbell, SL, Petzold, LR: Canonical forms and solvable singular systems of differential equations. SIAM J. Algebr. Discrete Methods 4, 517-521 (1983)

2. Rheinboldt, WC: Differential-algebraic systems as differential equations on manifolds. Math. Comput. 43(168), 473-482 (1984)

3. Chistyakov, VF, Shcheglova, AA: Izbrannye glavy teorii algebro-differentsialnykh sistem. (Selected chapters in the Theory of Differential-Algebraic Systems). Nauka, Novosibirsk (2003)

4. Brenan, KE, Campbell, SL, Petzold, LR: Numerical Solution of Initial-Boundary Problems in Differential-Algebraic Equations. Classics in Applied Mathematics, vol. 14. SIAM, Philadelphia (1996)

5. Samoilenko, AM, Shkil', MI, Yakovets, VP: Linear Systems of Differential Equations with Singularities. Vyshcha Shkola, Kiev (2000)

6. Boichuk, AA, Shegda, LM: Bifurcation of solutions of singular Fredholm boundary value problems. Differ. Equ. 47(4), 453-461 (2011)

7. Myshkis, AD, Samoilenko, AM: Systems with shocks at given moments of time. Mat. Sb. 74(2), 202-208 (1967) (in Russian)

8. Samoilenko, AM, Perestyuk, NA: Impulsive Differential Equations. Vyshcha Shkola, Kiev (1974) (in Russian)

9. Halanay, A, Wexler, D: Qualitative Theory of Impulsive Systems. Mir, Moscow (1971) (Russian translation)

10. Schwabik, S, Tvrdý, M, Vejvoda, O: Differential and Integral Equations: Boundary Value Problems and Adjoints. Academia and Reidel, Prague (1979)

11. Boichuk, AA, Samoilenko, AM: Generalized Inverse Operators and Fredholm Boundary-Value Problems. Brill, Utrecht (2004)

12. Samoilenko, AM, Boichuk, AA, Boichuk, AA: Solutions of weakly perturbed linear systems bounded on the entire axis. Ukr. Mat. Zh. 54, 1517-1530 (2002)

13. Boichuk, AA, Diblík, J, Khusainov, DY, Růžičková, M: Fredholm's boundary-value problems for differential systems with a single delay. Nonlinear Anal. 72, 2251-2258 (2010)

14. Zettl, A: Adjoint and self-adjoint boundary value problems with interface conditions. SIAM J. Appl. Math. 16(4), 851-859 (1968)

15. Boichuk, A, Pokutnij, A: Bounded solutions of linear perturbed differential equations in a Banach space. Tatra Mt. Math. Publ. 39, 1-12 (2007)

16. Boichuk, A, Langerová, M, Škoríková, J: Solutions of linear impulsive differential equations bounded on the whole line. Adv. Differ. Equ. 2010, Article ID 494379 (2010). doi:10.1155/2010/494379

17. Boichuk, A, Langerová, M, Škoríková, J: Existence conditions for bounded solutions of weakly perturbed linear impulsive systems. Abstr. Appl. Anal. 2011, Article ID 792689 (2011). doi:10.1155/2011/792689

doi:10.1186/1687-1847-2013-186

Cite this article as: Boichuk et al.: Systems of singular differential equations with pulse action. Advances in Difference Equations 2013 2013:186. 